\title{
Clinical and echocardiographic predictors of mortality in acute pulmonary embolism
}

Talal Dahhan ${ }^{1,6}$, Irfan Siddiqui ${ }^{2}$, Victor F. Tapson ${ }^{3}$, Eric J. Velazquez ${ }^{4}$, Stephanie Sun ${ }^{5}$, Clemontina A. Davenport ${ }^{5}$, Zainab Samad ${ }^{4}$ and Sudarshan Rajagopal ${ }^{4,6^{*}}$

\begin{abstract}
Purpose: The aim of this study was to evaluate the utility of adding quantitative assessments of cardiac function from echocardiography to clinical factors in predicting the outcome of patients with acute pulmonary embolism (PE).

Methods: Patients with a diagnosis of acute PE, based on a positive ventilation perfusion scan or computed tomography (CT) chest angiogram, were identified using the Duke University Hospital Database. Of these, 69 had echocardiograms within 24-48 h of the diagnosis that were suitable for offline analysis. Clinical features that were analyzed included age, gender, body mass index, vital signs and comorbidities. Echocardiographic parameters that were analyzed included left ventricular (LV) ejection fraction (EF), regional, free wall and global RV speckle-tracking strain, RV fraction area change (RVFAC), Tricuspid Annular Plane Systolic Excursion (TAPSE), pulmonary artery acceleration time (PAAT) and RV myocardial performance (Tei) index. Univariable and multivariable regression statistical analysis models were used.

Results: Out of 69 patients with acute PE, the median age was 55 and $48 \%$ were female. The median body mass index (BMI) was $27 \mathrm{~kg} / \mathrm{m}^{2}$. Twenty-nine percent of the cohort had a history of cancer, with a significant increase in cancer prevalence in non-survivors (57\% vs $29 \%, p=0.02$ ). Clinical parameters including heart rate, respiratory rate, troponin T level, active malignancy, hypertension and COPD were higher among non-survivors when compared to survivors ( $p \leq 0.05)$. Using univariable analysis, NYHA class III symptoms, hypoxemia on presentation, tachycardia, tachypnea, elevation in Troponin $\mathrm{T}$, absence of hypertension, active malignancy and chronic obstructive pulmonary disease (COPD) were increased in non-survivors compared to survivors $(p \leq 0.05)$. In multivariable models, RV Tei Index, global and free (lateral) wall RVLS were found to be negatively associated with survival probability after adjusting for age, gender and systolic blood pressure ( $p \leq 0.05)$.
\end{abstract}

Conclusion: The addition of echocardiographic assessment of RV function to clinical parameters improved the prediction of outcomes for patients with acute PE. Larger studies are needed to validate these findings.

Keywords: Echocardiography, Pulmonary embolism, Right ventricular function, Speckle-tracking echocardiography

\section{Background}

Acute pulmonary Embolism (PE) is a major cause of morbidity and mortality in the United States and Europe, accounting for 100,000 and 300,000 deaths annually, respectively $[1,2]$. PE can be classified as massive, submassive or nonmassive based on the hemodynamic

\footnotetext{
*Correspondence: sudarshan.rajagopal@duke.edu;

sudarshan.rajagopal@dm.duke.edu

${ }^{4}$ Department of Medicine, Division of Cardiology, Duke University, Durham, NC, USA

${ }^{6}$ Center for Pulmonary Vascular Disease, Box 102351, DUMC, Durham, NC 27710, USA

Full list of author information is available at the end of the article
}

status and right ventricular (RV) function of the patient. Massive PE is characterized by systemic hypotension or cardiogenic shock, submassive PE is characterized by RV dysfunction without hypotension, and nonmassive PE has neither systemic hypotension nor RV dysfunction [2]. Massive PE is associated with an in-hospital mortality of $25-50 \%$, submassive PE with a mortality rate of 3-15\%, while nonmassive PE is associated with mortality of $5 \%$ or less [3]. Risk assessment in patients with submassive PE can be difficult, as the mortality rates for submassive PE can approach that of massive PE [4]. While there is a consensus that systemic thrombolysis, 
catheter-directed interventions, or surgery are indicated in patients with massive PE, the management of patients with submassive PE remains controversial. Therefore, there remains a challenge in the clinical management of patients who have stable hemodynamics but demonstrate evidence of RV dysfunction, either by electrocardiogram, echocardiogram, computed tomography (CT) scan or cardiac biomarkers [2]. The benefit of thrombolytic or invasive therapies relative to the risk of bleeding is unclear among such patients [5]. An improved approach to risk assessment could therefore allow the identification of those patients presenting with submassive PE who would benefit most from therapy.

To address this, risk assessments based on clinical and imaging parameters have been developed. The Pulmonary Embolism Severity Index (PESI) is an excellent clinical predictor of outcomes in patients with PE [6]. It is based on 11 clinical criteria including age, sex, history of cancer and hemodynamic parameters. Five risk categories are included, ranging from very low risk, with 30-day mortality of less than $2 \%$, to very high risk, with $10.0-24.5 \%$ mortality [6]. The simplified PESI (sPESI) was subsequently developed, with only six, rather than 11 , clinical criteria. In this index, only two risk categories were included, with low risk associated with $1.1 \%$ mortality and high risk associated with an $8.9 \%$ risk of death [7]. Quantitative echocardiographic assessment has been gaining importance in patients with RV dysfunction, including those with congenital heart disease, pulmonary hypertension and pulmonary embolism $[8,9]$. A number of studies [10-21] have tested the utility of novel echocardiographic or serum biomarkers for risk assessment in acute PE, but only a few studies have tested whether such parameters provide additional benefit to clinical predictors [22, 23]. We hypothesized that the addition of quantitative echocardiographic markers of RV function would add to clinical parameters to predict outcomes in patients with acute PE.

\section{Methods}

\section{Study population}

We retrospectively identified patients who had a diagnosis of acute PE between January 2010 and April 2014, confirmed by contrasted computed tomography (CT) scan of the chest and/or ventilation-perfusion (VQ) nuclear medicine imaging at Duke University Medical Center (Durham, NC, USA) using the Duke Enterprise Data Unified Content Explorer (DEDUCE) [24]. Subjects were included in the study if they had an echocardiogram performed within 24-48 h of diagnosis of acute PE. Subjects were excluded if their echocardiographic images were suboptimal for RV strain measurement due to poor image quality or poor RV views. Clinical data including demographics, medical history, comorbidities, systemic blood pressure, heart rate, respiratory rate, oxygen saturation on room air and with supplemental oxygen, were collected from the medical record. The Duke University Medical Center Institutional Review Board approved this study.

\section{Echo-derived parameters of RV function}

All echocardiographic studies were performed on GE Vivid E9 using a $3.5 \mathrm{MHz}$ probe (GE, Vingmed Ultrasound, Hortom, Norway) or Philips IE33 (Philips, Netherlands). Off-line analyses of images were performed in Xcelera (Philips, Andover, MA) and ImageArena (TomTec Imaging Systems, Unterschleißheim, Germany) by a single experienced reader and analysis was confirmed by a separate experienced reader; interreader variability for these studies have been shown to be low [25]. TAPSE was determined from an M-mode through the lateral tricuspid annulus by calculating the amount of longitudinal motion of the annulus at peak systole [26] (Fig. 1a). RV Tei index was calculated as the $\mathrm{RV}$ isovolumic time (IVT) divided by the ejection time (ET) using the pulsed Doppler method [8] (Fig. 1c). IVT was calculated as the duration of tricuspid regurgitation from continuous wave Doppler across the tricuspid valve minus the ET from a single representative beat. ET was calculated as the duration of RV outflow on pulsed Doppler across the RVOT from a single representative beat (Fig. 1c). Care was taken to use beats with similar $R R$ intervals to minimize errors in calculation. RV Fraction Area Change (FAC) [27] was calculated as the [(RV end-diastolic area - end-systolic area)/end-diastolic area] $\times 100$ (Fig. 1d). The RV endocardium was traced in systole and diastole from the annulus, along the free wall, to the apex and back along the interventricular septum using the apical four-chamber view. Attempts were made to trace the free wall beneath trabeculations (Fig. 1d).

\section{RV longitudinal strain}

2D strain analysis was performed from the apical 4chamber view as previously described [28] (Fig. 1b). The reference point for a single cardiac cycle was placed at the beginning of the QRS. Pulmonic valve closure was determined from the pulsed wave Doppler profile of the RV outflow tract. The endocardial border was traced in end systole and the region of interest was adjusted to exclude the pericardium. The quality of the tracking was confirmed visually from $2 \mathrm{D}$ images and from the strain traces. Segments with persistently inadequate tracking despite attempts at improving border definition and region of interest were excluded from analysis. The longitudinal strain of the RV free wall $\left(\mathrm{RV}_{\text {free }}\right)$ was calculated as the average of the three free wall segments and the longitudinal strain of the RV septum $\left(R V_{\text {sept }}\right)$ was 


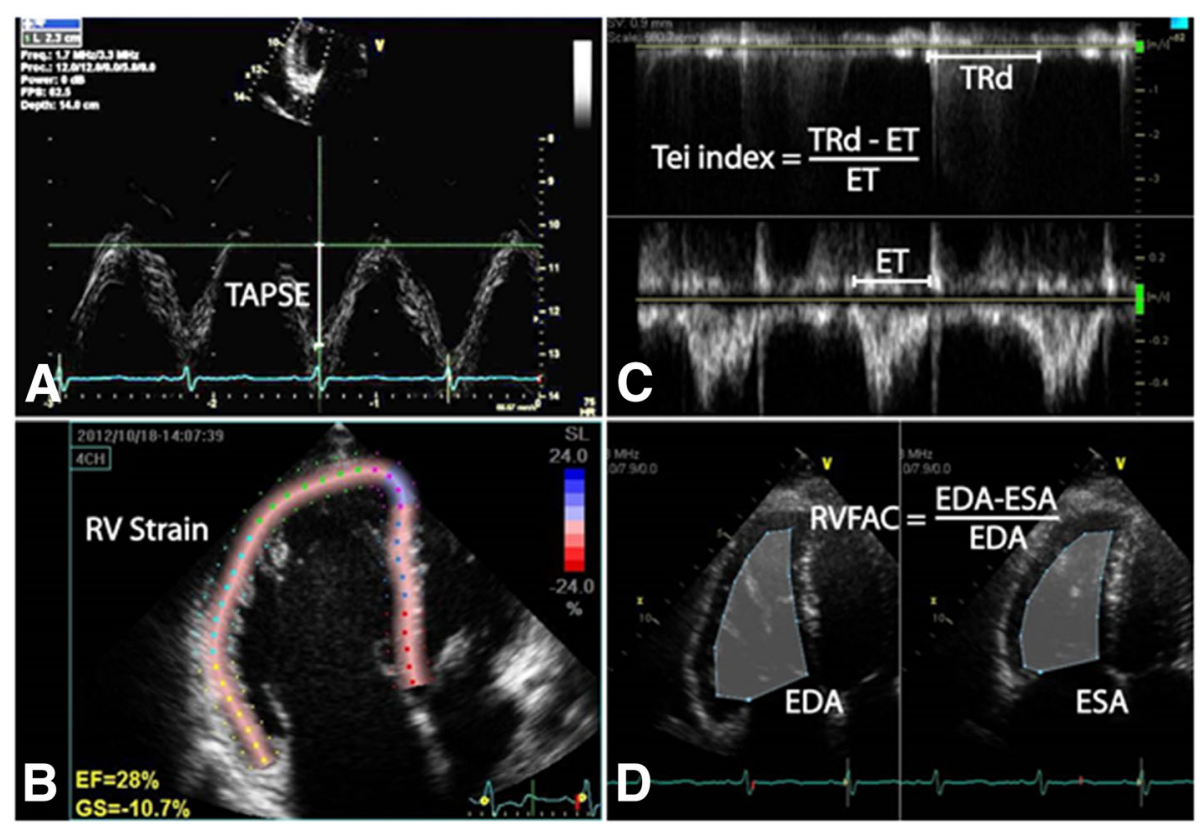

Fig. 1 Different 2D echocardiographic methods used in this study to assess RV function. a Tricuspid Annular Plane Systolic Excursion (TAPSE) is determined from an M-mode image through the lateral tricuspid annulus by calculating the amount of longitudinal motion of the tricuspid annulus at peak systole. $\mathbf{b}$ RV longitudinal strain is calculated from speckle-tracking of an RV focused apical 4-chamber view. c RV Tei index is calculated as the RV isovolumic time (equal to tricuspid regurgitation duration (TRd) - ejection time (ET)) divided by the ET using the pulsed Doppler method. $\mathbf{d}$ RV Fractional Area Change (RV FAC) was calculated as the [(RV end-diastolic area - end-systolic area)/end-diastolic area] $\times 100$

calculated as the average of the three septal segments. Global RVLS was calculated as the average of strains from all segments. All strain and other 2D echo-derived parameter analyses were performed blinded to clinical data.

\section{Statistical analysis}

Demographic and clinical characteristics of participants were presented in the study by survival status and the two groups were compared using Fisher's exact test for categorical variables and a Kruskal-Wallis rank sum test for continuous variables. Continuous variables were summarized by the median and interquartile range and categorical variables were summarized by counts and percentages (Tables 1 and 2). Table 3 displays the odds ratios and $95 \%$ confidence intervals resulting from univariable logistic regression modeling the probability of survival. These models investigated the association between clinical characteristics, cardiac biomarkers, specific echocardiographic features and PESI predictor score on the probability of survival and no adjustment for multiple testing was done. Multivariable models were fit to investigate the effects of some clinical features with echocardiographic parameters on survival, and the results are shown in Table 4. Statistical analyses were performed using SAS 9.4 (SAS, Cary, NC) and R (R Core Team (2015), Vienna, Austria).

\section{Results}

During the study period, 135 patients were admitted with a clinical diagnosis of acute PE. Among these, 95 patients diagnosed with an acute PE had a transthoracic echocardiogram within the initial $24-48 \mathrm{~h}$ of admission. 26 patients did not have suitable images for offline analysis, resulting in a cohort of 69 analyzed subjects. Six of the subjects underwent thrombolysis. At 30 days, of these 69 subjects, 14 had died and 55 survived. (Table 1).

\section{Baseline characteristics and presentation}

The baseline characteristics of all 69 patients are listed in Table 1. The median age was 55 years old (range 16-95) and $48 \%(n=38)$ of patients were females. The median body mass index (BMI) was $27 \mathrm{~kg} / \mathrm{m}^{2}$ (range 20-68). With respect to comorbidities, $29 \%(n=20)$ of the cohort had a history of cancer, with a significantly higher prevalence in non-survivors compared to survivors (57 \% vs. $29 \%$, $p=0.02)$. The only other significant difference in comorbidities between non-survivors and survivors was in the prevalence of hypertension (non-survivors, $64 \%$, vs. survivors, $25 \%, p=0.03) .13 \%$ of patients $(n=9)$ had a history of prior venous thromboembolism. On presentation, $90 \%(n=62)$ had NYHA class III symptoms (Table 1). Hypoxemia on presentation, tachycardia, tachypnea, elevation in Troponin $\mathrm{T}$, absence of hypertension, active malignancy and chronic 
Table 1 Baseline characteristics for the cohort of patients with acute PE

\begin{tabular}{|c|c|c|c|c|}
\hline \multirow[t]{2}{*}{ Parameter (n) } & All patients & Non-Survivors & Survivors & $p$-Value \\
\hline & 69 & 14 & 55 & \\
\hline Age at Diagnosis (69) & $55[43-72]$ & $60[53.5-69.8]$ & $50[42.5-72]$ & 0.11 \\
\hline Female Gender (69) & $33(48)$ & $6(42.86)$ & $27(49.09)$ & 0.77 \\
\hline Body Mass Index (69) & $27[23-28]$ & $26.5[22.2-27]$ & $27[23-28]$ & 0.42 \\
\hline \multicolumn{5}{|l|}{ Vital Signs: } \\
\hline Systolic Blood Pressure (66) & 118 [107-137] & $111[99.5-144.8]$ & 118 [109-136.5] & 0.38 \\
\hline Diastolic Blood Pressure (66) & $73[61.5-81.5]$ & $65[60.8-71]$ & $75.5[63.5-83.8]$ & 0.08 \\
\hline Heart Rate (66) & $94[84-111.5]$ & 115 [102.2-125.5] & $90[81.8-105.8]$ & 0.02 \\
\hline Respiratory Rate (66) & 20 [18-24] & $24[21.5-28]$ & 20 [18-24] & 0.01 \\
\hline Fraction of Inspired Oxygen (69) & $0.2[0.2-0.3]$ & $0.3[0.2-0.4]$ & $0.2[0.2-0.3]$ & 0.01 \\
\hline Troponin T level (ng/mL) (23) & $0.1[0-0.3]$ & $0.6[0.5-0.7]$ & $0.1[0-0.2]$ & $<0.01$ \\
\hline \multicolumn{5}{|l|}{ Medical History } \\
\hline Essential Hypertension (69) & $25(36.23)$ & $9(64.29)$ & $16(29.09)$ & 0.03 \\
\hline Type II Diabetes (69) & $4(5.8)$ & $1(7.14)$ & $3(5.45)$ & $>0.99$ \\
\hline Hypothyroidism (69) & $6(8.7)$ & $1(7.14)$ & $5(9.09)$ & $>0.99$ \\
\hline Chronic Kidney Disease (69) & $3(4.35)$ & $1(7.14)$ & $2(3.64)$ & 0.5 \\
\hline Previous Venous Thromboembolism (69) & $9(13.04)$ & $0(0)$ & $9(16.36)$ & 0.19 \\
\hline Connective Tissue Disease (69) & $2(2.9)$ & $1(7.14)$ & $1(1.82)$ & 0.37 \\
\hline Active Malignancy (69) & $20(28.99)$ & $8(57.14)$ & $12(21.82)$ & 0.02 \\
\hline Orthopedic Fracture or Injury (69) & $5(7.25)$ & $0(0)$ & $5(9.09)$ & 0.58 \\
\hline Chronic Obstructive Pulmonary Disease (69) & $5(7.25)$ & $3(21.43)$ & $2(3.64)$ & 0.05 \\
\hline \multicolumn{5}{|l|}{ Shortness of Breath: } \\
\hline NYHA class1 & $2(2.9)$ & $0(0)$ & $2(3.64)$ & \\
\hline NYHA class2 & $5(7.25)$ & $1(7.14)$ & $4(7.27)$ & \\
\hline NYHA class3 & $15(21.74)$ & $1(7.14)$ & $14(25.45)$ & \\
\hline NYHA class4 & $47(68.12)$ & $12(85.71)$ & $35(63.64)$ & \\
\hline Pulmonary Embolism Severity Index (PESI) class (69) & & & & $<0.01$ \\
\hline Class 1 & $32(46.38)$ & $1(7.14)$ & $31(56.36)$ & \\
\hline Class 2 & $10(14.49)$ & $2(14.29)$ & $8(14.55)$ & \\
\hline Class 3 & $17(24.64)$ & $5(35.71)$ & $12(21.82)$ & \\
\hline Class 4 & $10(14.49)$ & $6(42.86)$ & $4(7.27)$ & \\
\hline
\end{tabular}

Shown are median and inter-quartile range in brackets or number of patients with percent of patients in parentheses. $P$-value denotes comparison between nonsurvivors and survivors

Abbreviations: NYHA New York Heart Association

obstructive pulmonary disease (COPD) were statistically significantly different $(p \leq 0.05)$ between nonsurvivors and survivors (Table 1 ).

Echocardiographic assessment of RV function in acute PE A number of echocardiographic parameters were assessed in our cohort (Table 2). These included parameters that are thought to quantify RV systolic function (TAPSE, global, regional and free wall RV longitudinal strain (RVLS), RV myocardial performance (Tei) Index, RV fraction area change (RVFAC), and subjective echocardiographic evaluation of RV function) and RV size (RV/LV ratio in systole and diastole, diameters of RV and LV in systole and diastole). RVFAC, Tei Index, global and free wall RVLS were significantly different between survivors and nonsurvivors $(p \leq 0.05)$. For example, a significant proportion of non-survivors had global and free wall RVLS of more than -12.5 (Fig. 2), a value which has been demonstrated to be associated with worse outcomes in pulmonary hypertension [29]. TAPSE, subjective RV dilation and subjective RV dysfunction were not statistically different between survivors and nonsurvivors $(p>0.05)$. 
Table 2 Echocardiographic parameters in survivors and nonsurvivors with acute PE

\begin{tabular}{|c|c|c|c|c|}
\hline & Overall & Non-Survivors & Survivors & $p$-Value \\
\hline Parameter (n) & 69 & 14 & 55 & \\
\hline TAPSE (52) (cm) & $1.9[1.5-2.3]$ & $2.1[1.2-2.4]$ & $1.9[1.6-2.2]$ & 0.82 \\
\hline Global RVLS (69) (\%) & $-18.1[-20.8-14.9]$ & $-15.7[-19.2-12.1]$ & $-18.6[-21.8-15.6]$ & 0.05 \\
\hline Average Septal RV Wall strain (69) (\%) & $-17.8[-22.7-13.9]$ & $-16.8[-18.6-9.3]$ & $-17.8[-22.9-14.7]$ & 0.20 \\
\hline Average Free Wall RV Wall strain (69) (\%) & $-18.2[-22.8-13.9]$ & $-15[-18.7-10.3]$ & $-19.2[-23.2-14.3]$ & 0.04 \\
\hline RV Tei Index (59) & $0.5[0.4-0.5]$ & $0.5[0.5-0.6]$ & $0.5[0.4-0.5]$ & 0.01 \\
\hline Pulmonary Artery Acceleration Time $(64)(\mathrm{ms} \times 10)$ & $9.5[7-12]$ & $9[7-10]$ & $10[7-12]$ & 0.58 \\
\hline RV/LV ratio in systole (52) & $1[0.8-1.4]$ & $1.1[1-1.4]$ & $1[0.8-1.5]$ & 0.74 \\
\hline RV/LV ratio in diastole (52) & $0.7[0.6-0.9]$ & $0.8[0.7-1]$ & $0.7[0.6-0.9]$ & 0.35 \\
\hline RV base $(42)(\mathrm{cm})$ & $3.8[3.3-4.3]$ & $4.2[3.5-4.3]$ & $3.7[3.3-4.3]$ & 0.61 \\
\hline RV middle (39) (cm) & $3.3[2.9-3.6]$ & $3.5[3-4.5]$ & $3.3[2.9-3.6]$ & 0.31 \\
\hline RV length (39) (cm) & $7.5[7-7.9]$ & $7.5[7.2-7.8]$ & $7.5[6.9-7.9]$ & 0.61 \\
\hline RV diastolic diameter (52) (cm) & $2.7[2.1-3.1]$ & $2.7[2.3-3.1]$ & $2.7[2.1-3.2]$ & 0.41 \\
\hline LV diastolic diameter (52) (cm) & $3.5[2.9-4.1]$ & $3.2[2.9-3.8]$ & $3.6[3-4.2]$ & 0.63 \\
\hline RV systolic diameter (52) (cm) & $2.4[2.1-2.9]$ & $2.6[2.2-2.9]$ & $2.4[2-2.9]$ & 0.57 \\
\hline LV systolic diameter (34) (cm) & $2.3[1.9-2.7]$ & $2.2[1.9-3]$ & $2.4[2-2.6]$ & 0.65 \\
\hline RV fractional area change (67) (\%) & $34.4[27.6-43.1]$ & $27.9[19.6-35.8]$ & $38.6[28.9-43.8]$ & 0.02 \\
\hline RV area in diastole $(67)\left(\mathrm{cm}^{2}\right)$ & $24.3[19.9-31]$ & $25[22-44]$ & $24.1[19.3-29.8]$ & 0.22 \\
\hline RV area in Systole (67) $\left(\mathrm{cm}^{2}\right)$ & 16.8 [11.4-19.6] & $16.8[14.6-26.3]$ & $16.9[11.1-19.4]$ & 0.20 \\
\hline RV basal free wall strain (69) (\%) & $-21.4[-27.4-13.5]$ & $-14.8[-23.9-10.9]$ & $-22.4[-28.2-16.1]$ & 0.06 \\
\hline RV middle free wall strain (69) (\%) & $-16.3[-22.6-9.5]$ & $-12.4[-14.5-9.2]$ & $-17.9[-23.5-9.8]$ & 0.10 \\
\hline RV apical free wall strain (69) (\%) & $-13.9[-21.4-8.3]$ & $-11.4[-15.2-7.7]$ & $-14.9[-21.4-9.2]$ & 0.33 \\
\hline RV basal septal strain (69) (\%) & $-19[-24.5-13.9]$ & $-17.1[-20.1-13]$ & $-19.6[-24.8-13.9]$ & 0.23 \\
\hline RV middle septal strain (69) (\%) & $-18.4[-23.3-13.7]$ & $-15.6[-22.8-8.5]$ & $-19[-22.8-14.2]$ & 0.32 \\
\hline RV apical septal strain (69) (\%) & $-16[-19.7-9.5]$ & $-12.1[-17.7-7]$ & $-16.4[-19.9-9.8]$ & 0.15 \\
\hline RV subjective size & & & & 0.28 \\
\hline Normal RV size & $39(56.52)$ & $7(50)$ & $32(58.18)$ & \\
\hline Mild RV dilation & $11(15.94)$ & $3(21.43)$ & $8(14.55)$ & \\
\hline Moderate RV dilation & $12(17.39)$ & $1(7.14)$ & $11(20)$ & \\
\hline Severe RV dilation & $7(10.14)$ & $3(21.43)$ & $4(7.27)$ & \\
\hline RV subjective function & & & & 0.60 \\
\hline Normal RV function & $38(55.07)$ & $6(42.86)$ & $32(58.18)$ & \\
\hline Mildly reduced RV function & $10(14.49)$ & $2(14.29)$ & $8(14.55)$ & \\
\hline Moderately reduced RV function & $15(21.74)$ & $4(28.57)$ & $11(20)$ & \\
\hline Severely reduced RV function & $6(8.7)$ & $2(14.29)$ & $4(7.27)$ & \\
\hline LV ejection fraction (69) & & & & 0.07 \\
\hline LV ejection fraction < $40 \%$ & $6(8.7)$ & $3(21.43)$ & $3(5.45)$ & \\
\hline LV ejection fraction $40-50 \%$ & $6(8.7)$ & $2(14.29)$ & $4(7.27)$ & \\
\hline LV ejection fraction $>50 \%$ & $57(82.61)$ & $9(64.29)$ & $48(87.27)$ & \\
\hline
\end{tabular}

Shown are median and inter-quartile range in brackets or number of patients with percent of patients in parentheses. $P$-value denotes comparison between nonsurvivors and survivors

Abbreviations: $R V$ right ventricle, $L V$ left ventricle, TAPSE tricuspid annular plane systolic excursion, RVLS RV longitudinal strain 
Table 3 Univariable analysis of clinical and echocardiographic parameters in predicting outcome in acute PE

\begin{tabular}{|c|c|c|c|c|c|c|c|}
\hline & OR & $2.5 \%$ & $97.5 \%$ & Estimate & SE & Z & $p$-value \\
\hline Age at Diagnosis (69) (years) & 0.98 & 0.94 & 1.01 & -0.02 & 0.02 & -1.44 & 0.15 \\
\hline Female Gender (69) & 1.29 & 0.39 & 4.20 & 0.25 & 0.60 & 0.42 & 0.68 \\
\hline Body Mass Index (69) $\left(\mathrm{kg} / \mathrm{m}^{2}\right)$ & 1.06 & 0.93 & 1.20 & 0.06 & 0.06 & 0.87 & 0.39 \\
\hline Systolic blood pressure (66) (mmHg) & 1.01 & 0.99 & 1.04 & 0.01 & 0.01 & 0.91 & 0.36 \\
\hline Diastolic blood pressure (66) $(\mathrm{mmHg})$ & 1.04 & 1.00 & 1.10 & 0.04 & 0.02 & 1.80 & 0.07 \\
\hline Malignancy (69) & 0.21 & 0.06 & 0.72 & -1.56 & 0.63 & -2.48 & 0.01 \\
\hline Troponin T (23) (ng/mL) & 0.00 & 0.00 & 0.52 & -13.18 & 6.39 & -2.06 & 0.04 \\
\hline CK-MB (50) (ng/mL) & 0.99 & 0.93 & 1.04 & -0.01 & 0.03 & -0.52 & 0.60 \\
\hline Mild subjective RV dilation (69) & 0.58 & 0.12 & 2.77 & -0.54 & 0.80 & -0.68 & 0.50 \\
\hline Moderate subjective RV dilation (69) & 2.41 & 0.27 & 21.81 & 0.88 & 1.12 & 0.78 & 0.43 \\
\hline Severe subjective RV dilation (69) & 0.29 & 0.05 & 1.61 & -1.23 & 0.87 & -1.42 & 0.16 \\
\hline Mild subjective RV dysfunction (69) & 0.75 & 0.13 & 4.44 & -0.29 & 0.91 & -0.32 & 0.75 \\
\hline Moderate subjective RV dysfunction (69) & 0.52 & 0.12 & 2.17 & -0.66 & 0.73 & -0.90 & 0.37 \\
\hline Severe subjective RV dysfunction (69) & 0.37 & 0.06 & 2.53 & -0.98 & 0.97 & -1.01 & 0.31 \\
\hline LV ejection fraction $40-50 \%$ (69) & 2.00 & 0.19 & 20.61 & 0.69 & 1.19 & 0.58 & 0.56 \\
\hline LV ejection fraction > 50 \%(69) & 5.33 & 0.93 & 30.74 & 1.67 & 0.89 & 1.87 & 0.06 \\
\hline Global RVLS (69) (\%) & 0.88 & 0.78 & 0.99 & -0.13 & 0.06 & -2.13 & 0.03 \\
\hline Free wall RV strain (69) (\%) & 0.90 & 0.82 & 1.00 & -0.10 & 0.05 & -2.00 & 0.05 \\
\hline TAPSE (52) (cm) & 1.47 & 0.43 & 4.96 & 0.38 & 0.62 & 0.62 & 0.54 \\
\hline RV/LV ratio in systolic (52) & 1.10 & 0.23 & 5.22 & 0.10 & 0.79 & 0.12 & 0.90 \\
\hline RV/LV ratio in diastolic (52) & 0.39 & 0.02 & 6.50 & -0.95 & 1.44 & -0.66 & 0.51 \\
\hline RV Tei index (59) & 0.00 & 0.00 & 0.34 & -7.00 & 3.02 & -2.32 & 0.02 \\
\hline RVFAC (67) & 1.05 & 1.00 & 1.12 & 0.05 & 0.03 & 1.81 & 0.07 \\
\hline PAAT $(64)(m s \times 10)$ & 1.07 & 0.89 & 1.30 & 0.07 & 0.10 & 0.72 & 0.47 \\
\hline PESI class 3 (69) & 0.13 & 0.01 & 1.61 & -2.05 & 1.29 & -1.59 & 0.11 \\
\hline PESI class 4 (69) & 0.08 & 0.01 & 0.73 & -2.56 & 1.15 & -2.23 & 0.03 \\
\hline PESI class 5 (69) & 0.02 & 0.00 & 0.23 & -3.84 & 1.20 & -3.19 & $<0.01$ \\
\hline
\end{tabular}

Odds ratio (survival over non-survival), $95 \%$ confidence interval, estimate, standard error, and $p$-value of the univariable logistic regression models are shown Abbreviations: $C K-M B$, creatine kinase-myocardial band, $R V$ right ventricle, $L V$ left ventricle, RVLS RV longitudinal strain, TAPSE tricuspid annular plane systolic excursion, RVFAC RV fractional area change, PAAT pulmonary artery acceleration time, PESI pulmonary embolism severity index

\section{Univariable and multivariable predictors of outcome in acute PE}

On univariable analysis, a number of clinical and echocardiographic parameters were statistically significantly $(p \leq 0.05)$ associated with 30 day mortality. These included active malignancy, serum troponin, global and free wall RVLS, RV Tei Index, and patients with PESI classes four and five. For multivariable regression analysis, we attempted to include PESI as a clinical predictor with selected echo parameters, but could not because PESI score (whether categorical or continuous) demonstrated non-trivial collinearity with global and free wall RVLS and RV Tei index, resulting in unstable standard errors of estimates. Similarly, both heart rate and systolic blood pressure displayed significant collinearity, so only systolic blood pressure was included as it was a predictor in the univariable model. As the multivariable models could not include those clinical and echocardiographic predictors together, we instead used a multivariable model that included parameters used to calculate PESI [6], namely age, gender and systolic blood pressure. With this multivariable regression model, global and free wall RVLS and RV Tei index all predicted outcome with statistical significance $(p \leq 0.05)$.

\section{Discussion}

This study demonstrates that the addition of selected echocardiographic estimates of RV function to clinical parameters in patients with acute PE improved prediction of 30-day mortality in a cohort of patients with acute PE. In our cohort, global, free wall RVLS and RV Tei index analyses were independently associated with mortality on univariable and multivariable analysis. However, other assessments of RV function, including 
Table 4 Multivariable analysis of clinical and echocardiographic parameters in predicting outcome in acute PE

\begin{tabular}{|c|c|c|c|c|c|c|c|}
\hline & OR & $2.5 \%$ & $97.5 \%$ & Estimate & SE & Z & $p$-value \\
\hline \multicolumn{8}{|l|}{ Global RVLS model } \\
\hline Age & 0.99 & 0.95 & 1.02 & -0.01 & 0.02 & -0.74 & 0.46 \\
\hline Female Gender & 1.20 & 0.32 & 4.58 & 0.18 & 0.68 & 0.27 & 0.79 \\
\hline Systolic Blood Pressure & 1.01 & 0.99 & 1.04 & 0.01 & 0.01 & 0.97 & 0.33 \\
\hline Global RVLS & 0.87 & 0.77 & 1.00 & -0.14 & 0.07 & -2.01 & 0.04 \\
\hline \multicolumn{8}{|l|}{ Free wall RVLS model } \\
\hline Age & 0.99 & 0.95 & 1.03 & -0.01 & 0.02 & -0.68 & 0.50 \\
\hline Female Gender & 1.21 & 0.32 & 4.61 & 0.19 & 0.68 & 0.28 & 0.78 \\
\hline Systolic Blood Pressure & 1.01 & 0.98 & 1.04 & 0.01 & 0.01 & 0.67 & 0.50 \\
\hline Free wall RVLS & 0.89 & 0.80 & 1.00 & -0.11 & 0.06 & -1.95 & 0.05 \\
\hline \multicolumn{8}{|l|}{ RV Tei Index model } \\
\hline Age at Diagnosis & 0.99 & 0.95 & 1.03 & -0.01 & 0.02 & -0.53 & 0.60 \\
\hline Female Gender & 0.78 & 0.17 & 3.47 & -0.26 & 0.76 & -0.33 & 0.74 \\
\hline Systolic Blood Pressure & 1.02 & 0.99 & 1.05 & 0.02 & 0.02 & 1.19 & 0.23 \\
\hline RV Tei Index & 0.00 & 0.00 & 0.36 & -7.82 & 3.46 & -2.26 & 0.02 \\
\hline
\end{tabular}

TAPSE, RVFAC, and subjective evaluations of RV size and function were not associated with mortality on univariable analysis. At this time, there are no clear guidelines as to which parameters should be used to assess RV function [8], and significant inter-rater variability exists in subjective evaluation of the RV [9]. The objective echocardiographic assessment of RV function with

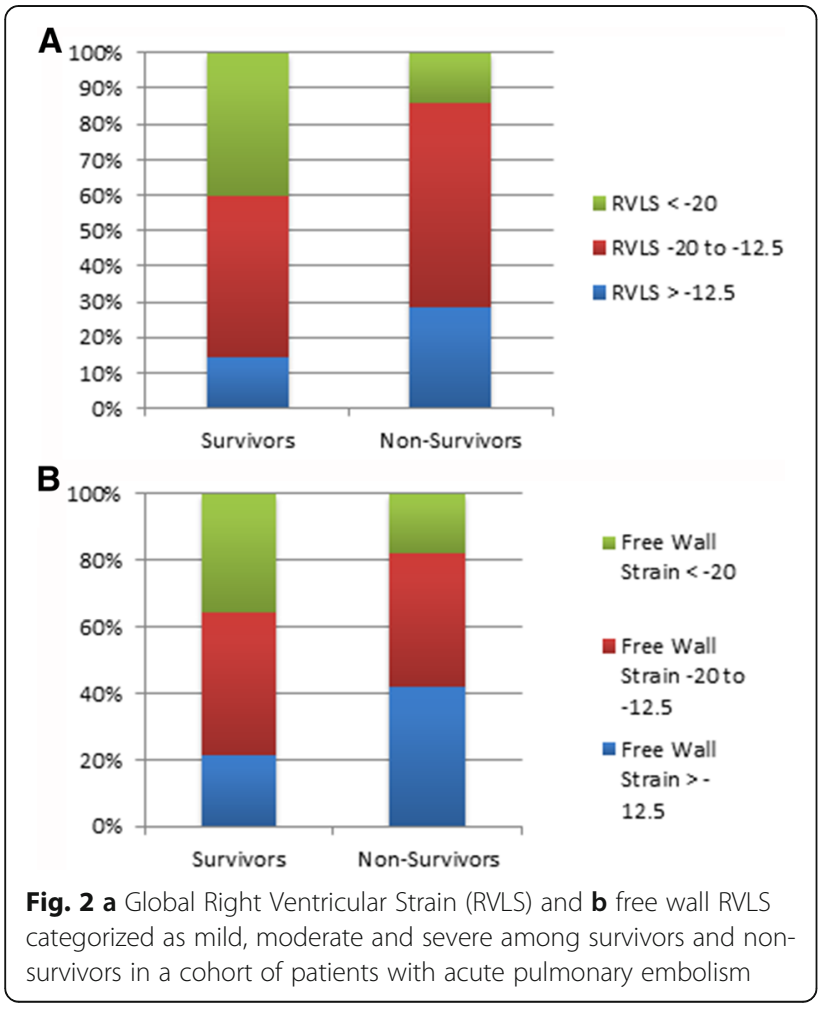

qualitative parameters, such as RVLS, may reduce interrater variability [25] and have utility in identifying submassive PE patients who may benefit most from consideration of aggressive therapies.

The European Society of Cardiology [2] and the American College of Chest Physicians guidelines [30] emphasize the importance of the assessment of RV function and cardiac biomarkers in risk assessment of acute PE, as they may allow the identification of high-risk patients before they clinically deteriorate. An alternative strategy has been the use of clinical risk prediction algorithms, such as PESI and SPESI $[6,7]$. In our analysis, we found significant collinearity between PESI and RVLS and Tei index, suggesting that these echocardiographic and clinical parameters are all associated with high risk features. While global and free wall RVLS require special software and analysis to obtain, RV Tei index is relatively straightforward to acquire and could be used broadly. Vitarelli et al. found an association of a number of parameters of RV function with 6 month adverse outcomes in acute PE patients on univariate analysis [27]. Moreover, they found that mid-free wall RVLS, RVSP and 3D RV ejection fraction were associated with adverse outcome on multivariate analysis. While we observed large absolute numerical differences in basal free wall strain between survivors and nonsurvivors, they did not reach statistical significance due to large variance. It is likely that they would have been significant in a larger study. Overall, the results here extend the findings of Vitarelli et al., as we found that both global and free wall RVLS were associated with outcome on multivariable analysis after accounting for age, gender and systolic blood pressure. 
While subjective RV dysfunction has been associated with worse outcomes in PE [31], a number of studies suggest that quantifiers of RV function may better identify high-risk patients, although most of these studies did not test the utility of such parameters in combination with clinical characteristics [11, 13-21]. For example, RV dysfunction, as assessed by tricuspid annular plane systolic excursion (TAPSE) and RV myocardial performance (Tei) index, has been characterized in patients with PE [32, 33]. Another recent study identified the ratio of RV to LV end-diastolic diameter, RV systolic pressure, tricuspid annular plane systolic excursion, and inferior vena cava collapsibility to be independently associated with mortality in patients presenting with acute PE [11]. Abnormal RV global and free wall speckle-tracking strains have been associated with adverse events in patients with PE [27]. Ozsu and coworkers demonstrated a correlation of Tei index with treatment response in acute PE [33]. Park and coworkers demonstrated that TAPSE correlated with other parameters of RV function and BNP in acute PE [32]. RV ejection fraction and regional mid wall strain has been prospectively assessed in patients with $\mathrm{PE}$, with a potential to assess a therapeutic response [27]. Thus, there are a number of parameters of RV function that have been shown to correlate with outcomes in acute PE.

\section{Conclusions}

Although our cohort of patients is small, we found an association of free-wall, global RV strain and RV Tei index with mortality. Notably, we did not find such a relationship between other measures, including TAPSE, $\mathrm{RV}$ size and RV/LV ratio. The associations we found were still significant after the inclusion of clinical risk factors $[6,7]$. These findings suggest that the addition of echocardiographic parameters to clinical parameters may improve risk prediction in acute PE.

\section{Limitations}

Our study is a retrospective, single center study with a small cohort of patients, which may limit the generalizability of these findings. Patient care was variable, resulting in significant differences in studies that were performed, such as troponin $\mathrm{T}$, which was only performed in 23 of the 69 subjects included in the final analysis. As there was no set protocol for RV imaging in this retrospective study, there was a relatively low suitability $(72.6 \%)$ of images for offline analysis and poor tracking observed in the basal and mid-free wall segments. In our analysis, we found significant collinearity between PESI and RVLS and Tei index, preventing us from combining our echo predictors with PESI score. Therefore, it is possible that echo predictors do not add significant information to the PESI score. Previous studies from our group have demonstrated small interand intra-observer variability in assessment of RV strain [34], so such analyses were not included in this study. Future prospective, multi-center studies that address objective RV function assessment in relation to outcomes in patients with PE are needed to validate these findings.

\begin{abstract}
Abbreviations
2D: Two dimensional; 3D: Three dimensional; BNP: Brain natriuretic peptide; COPD: Chronic obstructive pulmonary disease; CT: Computed tomography; DEDUCE: Duke Enterprise Data Unified Content Explorer; EF: Ejection fraction; ET: Ejection time; GE: General electric; IVT: Iso volumic time; LV: Left ventricle; PAAT: Pulmonary artery acceleration time; PE: Pulmonary embolism; PESI: Pulmonary embolism severity index; RV: Right ventricle; RVFAC: Right ventricular fraction area change; RVLS: Right ventricular longitudinal strain; RVOT: Right ventricular outflow tract; SAS: Statistical analysis system software; SPESI: simplified pulmonary embolism severity index; TAPSE: Tricuspid annular plane systolic excursion; VQ: Ventilation perfusion
\end{abstract}

Ethics approval and consent to participate

This study was approved by the institutional review board at Duke University. All the data are available as stored within a secure drive that is shared the among study authors.

\section{Competing interests}

The authors declare that they have no competing interests.

\section{Funding}

Funding was obtained from NIH grants K08HL114643, Gilead Research Scholars in Pulmonary Arterial Hypertension and a Burroughs Welcome Career Award for Medical Scientists supporting Sudarshan Rajagopal, the corresponding author. Clemontina Davenport is partially supported by Duke CTSA grant UL1TR001117.

\section{Authors' contributions}

TD, ZS and SR designed the study. TD collected data and wrote the manuscript. IS collected data, performed the echocardiographic analysis. SS and CD performed the statistical analysis. VT, EV, ZS and SR reviewed and edited the manuscript. All authors read and approved the final manuscript.

\section{Author details}

${ }^{1}$ Department of Medicine, Division of Pulmonary, Allergy and Critical Care Medicine, Duke University, Durham, NC, USA. ²Department of Medicine, East Carolina University, Greenville, NC, USA. ${ }^{3}$ Department of Medicine, Division of Pulmonary and Critical Care Medicine, Cedars Sinai Medical Center, Los Angeles, CA, USA. ${ }^{4}$ Department of Medicine, Division of Cardiology, Duke University, Durham, NC, USA. ${ }^{5}$ Department of Biostatistics and Bioinformatics, Duke University Medical Center, Durham, NC, USA. ${ }^{6}$ Center for Pulmonary Vascular Disease, Box 102351, DUMC, Durham, NC 27710, USA.

Received: 22 August 2016 Accepted: 19 October 2016

Published online: 28 October 2016

\section{References}

1. Horlander KT, Mannino DM, Leeper KV. Pulmonary embolism mortality in the United States, 1979-1998: an analysis using multiple-cause mortality data. Arch Intern Med. 2003;163(14):1711-7.

2. Konstantinides SV, Torbicki A, Agnelli G, et al. 2014 ESC guidelines on the diagnosis and management of acute pulmonary embolism. Eur Heart J. 2014;35(43):3033-69. 3069a-3069k.

3. Torbicki A, Perrier A, Konstantinides S, et al. Guidelines on the diagnosis and management of acute pulmonary embolism: the Task Force for the Diagnosis and Management of Acute Pulmonary Embolism of the European Society of Cardiology (ESC). Eur Heart J. 2008;29(18):2276-315.

4. Goldhaber SZ, Visani L, De Rosa M. Acute pulmonary embolism: clinical outcomes in the International Cooperative Pulmonary Embolism Registry (ICOPER). Lancet. 1999;353(9162):1386-9.

5. Meyer G, Vicaut E, Danays T, et al. Fibrinolysis for patients with intermediate-risk pulmonary embolism. N Engl J Med. 2014;370(15):1402-11. 
6. Aujesky D, Obrosky DS, Stone RA, et al. Derivation and validation of a prognostic model for pulmonary embolism. Am J Respir Crit Care Med. 2005;172(8):1041-6.

7. Jimenez D, Aujesky D, Moores $L$, et al. Simplification of the pulmonary embolism severity index for prognostication in patients with acute symptomatic pulmonary embolism. Arch Intern Med. 2010;170(15):1383-9.

8. Rudski LG, Lai WW, Afilalo J, et al. Guidelines for the echocardiographic assessment of the right heart in adults: a report from the American Society of Echocardiography endorsed by the European Association of Echocardiography, a registered branch of the European Society of Cardiology, and the Canadian Society of Echocardiography. J Am Soc Echocardiogr. 2010;23(7):685-713. quiz 786-688.

9. Lang RM, Bierig M, Devereux RB, et al. Recommendations for chamber quantification: a report from the American Society of Echocardiography's Guidelines and Standards Committee and the Chamber Quantification Writing Group, developed in conjunction with the European Association of Echocardiography, a branch of the European Society of Cardiology. J Am Soc Echocardiogr. 2005;18(12):1440-63.

10. Dursunoglu N, Dursunoglu D, Yildiz Al, Rota S. Evaluation of cardiac biomarkers and right ventricular dysfunction in patients with acute pulmonary embolism. Anadolu Kardiyol Derg. 2014.

11. Khemasuwan D, Yingchoncharoen T, Tunsupon $P$, et al. Right ventricular echocardiographic parameters are associated with mortality after acute pulmonary embolism. J Am Soc Echocardiogr. 2015;28(3):355-62.

12. Weekes AJ, Thacker G, Troha D, et al. Diagnostic accuracy of right ventricular dysfunction markers in normotensive emergency department patients with acute pulmonary embolism. Ann Emerg Med. 2016.

13. George E, Kumamaru KK, Ghosh N, et al. Computed tomography and echocardiography in patients with acute pulmonary embolism: part 2: prognostic value. J Thorac Imaging. 2014;29(1):W7-12.

14. Lee $\mathrm{JH}$, Park $\mathrm{JH}$, Park Kl, et al. A comparison of different techniques of twodimensional speckle-tracking strain measurements of right ventricular systolic function in patients with acute pulmonary embolism. J Cardiovasc Ultrasound. 2014;22(2):65-71.

15. Lobo JL, Holley A, Tapson V, et al. Prognostic significance of tricuspid annular displacement in normotensive patients with acute symptomatic pulmonary embolism. J Thromb Haemost. 2014;12(7):1020-7.

16. Platz E, Hassanein AH, Shah A, Goldhaber SZ, Solomon SD. Regional right ventricular strain pattern in patients with acute pulmonary embolism. Echocardiography. 2012;29(4):464-70.

17. Pruszczyk P, Goliszek S, Lichodziejewska B, et al. Prognostic value of echocardiography in normotensive patients with acute pulmonary embolism. JACC Cardiovasc Imaging. 2014;7(6):553-60.

18. Rodrigues AC, Cordovil A, Monaco C, et al. Right ventricular assessment by tissue-Doppler echocardiography in acute pulmonary embolism. Arq Bras Cardiol. 2013;100(6):524-30

19. Rodrigues AC, Cordovil A, Monaco CG, et al. Assessing prognosis of pulmonary embolism using tissue-Doppler echocardiography and brain natriuretic peptide. Einstein (Sao Paulo). 2013;11(3):338-44.

20. Rydman R, Soderberg M, Larsen F, Caidahl K, Alam M. Echocardiographic evaluation of right ventricular function in patients with acute pulmonary embolism: a study using tricuspid annular motion. Echocardiography. 2010; 27(3):286-93.

21. Stergiopoulos K, Bahrainy S, Strachan P, Kort S. Right ventricular strain rate predicts clinical outcomes in patients with acute pulmonary embolism. Acute Card Care. 2011;13(3):181-8.

22. Vitarelli A, Barilla F, Capotosto L, et al. Right Ventricular Function in Acute Pulmonary Embolism: A Combined Assessment by Three-Dimensional and Speckle-Tracking Echocardiography. J Am Soc Echocardiogr. 2014;27(3):329-38.

23. Pruszczyk P, Goliszek S, Lichodziejewska B, et al. Prognostic Value of Echocardiography in Normotensive Patients With Acute Pulmonary Embolism. JACC Cardiovasc Imaging. 2014.

24. Horvath MM, Winfield S, Evans S, Slopek S, Shang H, Ferranti J. The DEDUCE Guided Query tool: providing simplified access to clinical data for research and quality improvement. J Biomed Inform. 2011;44(2):266-76.

25. Forsha DE, Risum N, Smith PB, et al. A Novel Comprehensive RV Strain Analysis: Echocardiographic Approach to Define a Normal Adult Population. J Am Soc Echocardiogr. 2014:In press.

26. Kaul S, Tei C, Hopkins JM, Shah PM. Assessment of right ventricular function using two-dimensional echocardiography. Am Heart J. 1984;107(3):526-31.
27. Vitarelli A, Barilla F, Capotosto L, et al. Right ventricular function in acute pulmonary embolism: a combined assessment by three-dimensional and speckle-tracking echocardiography. J Am Soc Echocardiogr. 2014;27(3):329-38.

28. Risum N, Jons C, Olsen NT, et al. Simple regional strain pattern analysis to predict response to cardiac resynchronization therapy: rationale, initial results, and advantages. Am Heart J. 2012;163(4):697-704.

29. Sachdev A, Villarraga HR, Frantz RP, et al. Right ventricular strain for prediction of survival in patients with pulmonary arterial hypertension. Chest. 2011;139(6):1299-309.

30. Guyatt GH, Norris SL, Schulman S, et al. Methodology for the development of antithrombotic therapy and prevention of thrombosis guidelines: Antithrombotic Therapy and Prevention of Thrombosis, 9th ed: American College of Chest Physicians Evidence-Based Clinical Practice Guidelines. Chest. 2012;141(2 Suppl):53s-70.

31. McConnell MV, Solomon SD, Rayan ME, Come PC, Goldhaber SZ, Lee RT. Regional right ventricular dysfunction detected by echocardiography in acute pulmonary embolism. Am J Cardiol. 1996;78(4):469-73.

32. Park JH, Kim JH, Lee JH, Choi SW, Jeong JO, Seong IW. Evaluation of right ventricular systolic function by the analysis of tricuspid annular motion in patients with acute pulmonary embolism. J Cardiovasc Ultrasound. 2012; 20(4):181-8.

33. Ozsu S, Kiris A, Bulbul Y, et al. Relationship between cardiac troponin-T and right ventricular Tei index in patients with hemodynamically stable pulmonary embolism: an observational study. Anadolu Kardiyol Derg. 2012; 12(8):659-65.

34. Forsha D, Risum N, Kropf PA, et al. Right ventricular mechanics using a novel comprehensive three-view echocardiographic strain analysis in a normal population. J Am Soc Echocardiogr. 2014;27(4):413-22.

\section{Submit your next manuscript to BioMed Central and we will help you at every step:}

- We accept pre-submission inquiries

- Our selector tool helps you to find the most relevant journal

- We provide round the clock customer support

- Convenient online submission

- Thorough peer review

- Inclusion in PubMed and all major indexing services

- Maximum visibility for your research

Submit your manuscript at www.biomedcentral.com/submit
Biomed Central 